Article

\title{
Direct Conversion of Carbohydrates into Ethyl Levulinate with Potassium Phosphotungstate as an Efficient Catalyst
}

\section{Shiqiang Zhao ${ }^{1}$, Guizhuan Xu ${ }^{2, *}$, Chun Chang ${ }^{1, *}$, Shuqi Fang ${ }^{1}$, Ze Liu ${ }^{1}$ and Fengguang Du ${ }^{3}$}

1 School of Chemical Engineering and Energy, Zhengzhou University, Science Road 100, Zhengzhou 450001, China; E-Mails: sqzhao@yeah.net (S.Z.); fangsq@zzu.edu.cn (S.F.); liuze.666@163.com (Z.L.)

2 College of Mechanical and Electrical Engineering, Henan Agricultural University, Wenhua Road 95, Zhengzhou 450002, China

3 State Key Laboratory of Motor Vehicle Biofuel Technology, Nanyang 473000, China; E-Mail: dufengguang@163.com

* Authors to whom correspondence should be addressed; E-Mails: xuguizhuan@126.com (G.X.); chunchang@zzu.edu.cn (C.C.); Tel.: +86-371-136-5386-0867 (G.X.); +86-371-67780093 (C.C.).

Academic Editor: Rafael Luque

Received: 18 September 2015 / Accepted: 5 November 2015 / Published: 12 November 2015

\begin{abstract}
A series of metal-modified phosphotungstates were prepared and performed for direct synthesis of ethyl levulinate from fructose in ethanol. Considering the cost of catalysts, catalytic activity of catalysts, and easy separation of catalysts together, K-HPW-1 was chosen as the most suitable catalyst for synthesis of ethyl levulinate from fructose. A high ethyl levulinate yield of $64.6 \mathrm{~mol} \%$ was obtained at $150{ }^{\circ} \mathrm{C}$ within $2 \mathrm{~h}$ in ethanol. The introduction of low polar toluene as a co-solvent improved the yield of ethyl levulinate to $68.7 \mathrm{~mol} \%$. The recovered catalyst remained high activity with the yield of ethyl levulinate converted from fructose above $50 \mathrm{~mol} \%$ after being used five times. Moreover, the generality of the catalyst was further demonstrated by glucose, sucrose, inulin, and cellulose with ethyl levulinate yielding $14.5,35.4,52.3$, and $14.8 \mathrm{~mol} \%$, respectively.
\end{abstract}

Keywords: ethyl levulinate; carbohydrates; potassium phosphotungstate 


\section{Introduction}

With the gradual diminishment of fossil fuel reserves and increased environmental concerns, it is quite necessary to develop and utilize renewable resources. Carbohydrates derived from biomass are one of the most abundant renewable resources and possess a great potential as raw materials to product fuels and bulk chemicals [1,2]. Among those bio-based products, levulinic acid has been listed as one of the 12 most important building blocks, which can be used for the production of various high-value organic chemicals, such as resin, polymers, pharmaceutical compounds, and flavor substances [3-5]. Therefore, much effort has been devoted to the research for the synthesis of levulinic acid from carbohydrates in the past decades [6-10].

In the recent years, there has been growing interest in the synthesis levulinate esters including methyl, ethyl, and $n$-butyl levulinate. Among these, ethyl levulinate, obtained by esterification of levulinic acid, is a versatile chemical compound and has been widely applied in food industry, as solvents and plasticizers. Besides, it can be used as an additive for transportation fuels, which shows excellent performance $[11,12]$. It is reported that furfuryl alcohol also can be converted into ethyl levulinate in the presence of an acid catalyst [13]. However, both levulinic acid and furfuryl alcohol need to be prepared from carbohydrates or biomass firstly, and then need to be purified for synthesis of ethyl levulinate. In addition, there is a third route for synthesis of ethyl levulinate that is to be converted from carbohydrates or biomass directly [14-17]. This route provides a convenient one-pot reaction to produce ethyl levulinate. Compared with the former two routes, this route has some advantages, like easy purification, minimized wastewater, and time saving [18]. The three reaction routes are shown in Figure 1.

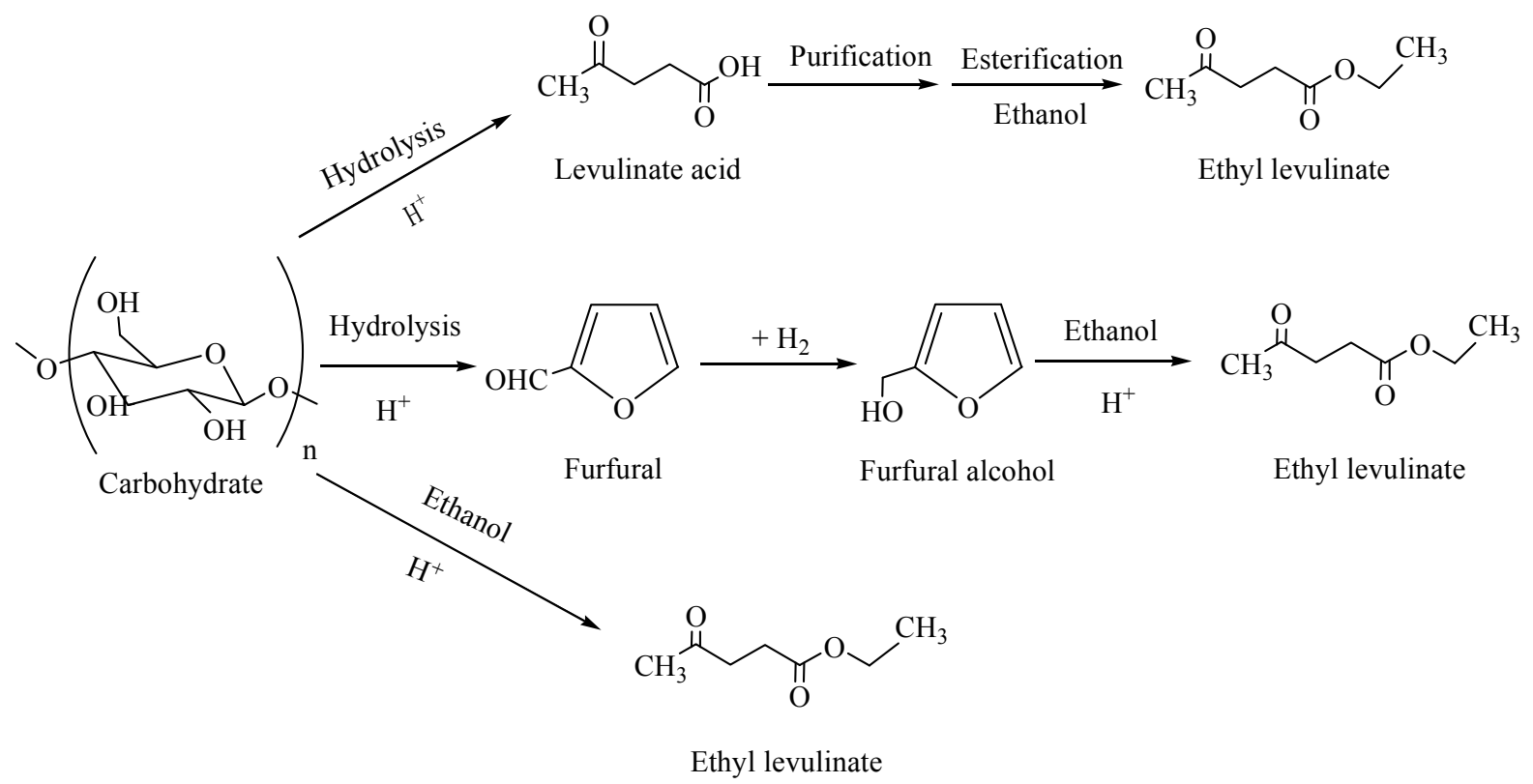

Figure 1. The routes of synthesis of ethyl levulinate.

To improve the direct synthesis of ethyl levulinate from carbohydrates derived from biomass, efficient acid catalysts have been developed, especially heterogeneous acid catalysts. Among various heterogeneous catalysts, Keggin-type heteropolyacid $\mathrm{H}_{3} \mathrm{PW}_{12} \mathrm{O}_{40}$ (HPW) with a strong Brønsted acidity has attracted much attention [19], but its application was limited due to the high solubility in water and 
polar solvents. The substitution of $\mathrm{H}^{+}$of $\mathrm{HPW}$ by large monovalent cations such as $\mathrm{Cs}^{+}, \mathrm{NH}_{4}^{+}, \mathrm{K}^{+}$and $\mathrm{Ag}^{+}$could modify the acidity of HPW and form a range of insoluble solid acid catalysts. Currently, little research on levulinate production from carbohydrates catalyzed by metal-modified phosphotungstates has been reported. Liu has reported that fructose can be efficiently converted into methyl levulinate with the catalysis of Fe-HPW-1, and the methyl levulinate yield of $73.7 \%$ was obtained at $130{ }^{\circ} \mathrm{C}$ for $2 \mathrm{~h}[20]$. However, Fe-HPW-1 is soluble in the methanol medium, and has to be extracted by diethyl ether for the next use. Herein, it is necessary to develop metal-modified phosphotungstates with high catalytic activity and easy recovery.

In this study, various metal-modified phosphotungstates were prepared to convert ethyl levulinate from fructose in ethanol medium. Among these catalysts, insoluble K-HPW-1 showed highest catalytic activity for direct conversion of fructose into ethyl levulinate with a yield of $64.6 \mathrm{~mol} \%$. Meanwhile, toluene as a co-solvent showed a good performance on direct synthesis of ethyl levulinate from fructose. Moreover, the catalytic system was extended to various carbohydrates. This recycling study showed that K-HPW-1 relative to HPW could be facile to separate from reaction mixture by centrifugation and be reused with good catalytic activity.

\section{Results and Discussion}

\subsection{Synthesis of Ethyl Levulinate from Fructose over Various Catalysts}

A series of metal-modified phosphotungstates prepared were firstly performed for direct synthesis of ethyl levulinate from fructose in ethanol, and the results are presented in Figure 2. It was reported that insoluble Cs-HPW-2.5 and Cs-HPW-1 have porous structures, relatively high surface areas, and strong acid sites [21], but they did not show good performance for alcoholysis of fructose to ethyl levulinate. The yield of ethyl levulinate decreased with $\mathrm{K}^{+}$instead of $\mathrm{H}^{+}$in HPW gradually, and this is due to the decrease of Brønsted acidity of potassium phosphotungstates. Compared with other prepared phosphotungstates, Ag-HPW-1, Fe-HPW-1, and K-HPW-1 showed high catalytic activity in terms of synthesis of ethyl levulinate from fructose with yield of 58.1, 52.4, and $50.8 \mathrm{~mol} \%$, respectively. However, it was found that Fe-HPW-1 can be dissolved in ethanol, and high volatile and flammable diethyl ether has to be used to recover it [20]. Although ethyl levulinate yield obtained catalyzed by Ag-HPW-1 was a little higher than that of K-HPW-1, $\mathrm{AgNO}_{3}$ that was used to synthesize Ag-HPW-1 was much more expensive than $\mathrm{KCl}$ used to synthesize $\mathrm{K}-\mathrm{HPW}-1$. Moreover, homogeneous $\mathrm{HPW}$ showed the highest activity and remarkably highest selectivity with ethyl levulinate yield of 64.9 mol\%. But, HPW is soluble in ethanol medium and is not easy to separate from ethanol. Thus, considering the cost of catalyst, yield of ethyl levulinate, and easy separation of catalyst together, K-HPW-1 was chosen as the suitable catalyst for synthesis of ethyl levulinate from fructose in the subsequent exploration. 


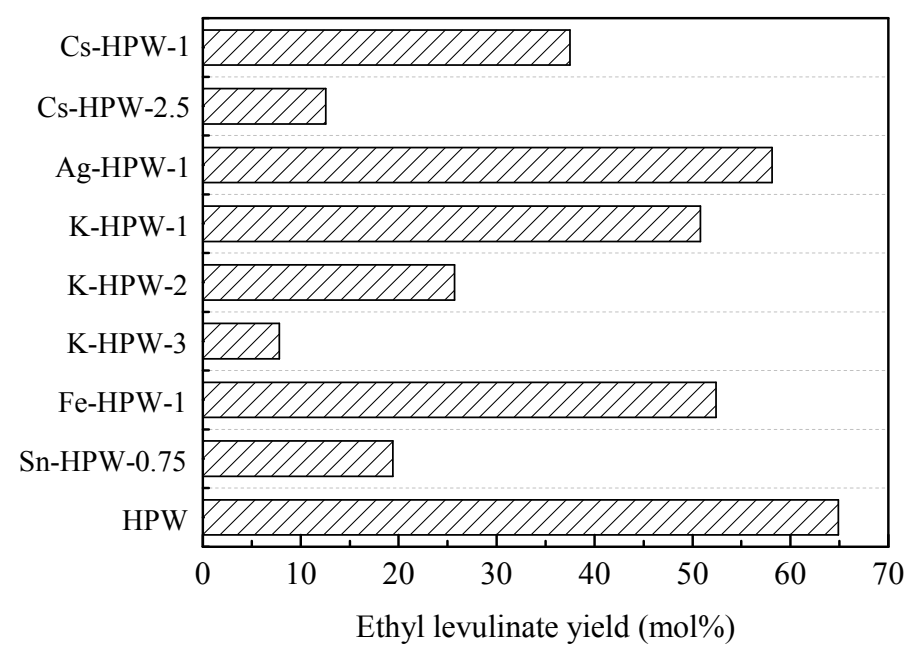

Figure 2. Effect of various catalysts on ethyl levulinate yield. Reaction conditions: fructose $0.8 \mathrm{~g}$, catalyst $0.4 \mathrm{~g}$, ethanol $40 \mathrm{~mL}$, temperature $130{ }^{\circ} \mathrm{C}$, reaction time $2 \mathrm{~h}$.

\subsection{Catalyst Characterization}

XRD patterns of HPW, K-HPW-1, K-HPW-2, and K-HPW-3 are displayed in Figure 3a. HPW showed typical cubic secondary structure of Keggin anion with characteristic diffraction peaks at $10.2^{\circ}$, $21.3^{\circ}, 25.3^{\circ}$, and $34.4^{\circ}$ [22]. K-HPW-1, K-HPW-2, and K-HPW-3 showed similar XRD patterns to that of HPW and present cubic secondary structure. The results indicated that Keggin structure was not destroyed during catalyst preparation in case of introduction of potassium. Moreover, the intensity of diffraction peaks decreased with the addition of potassium in HPW.
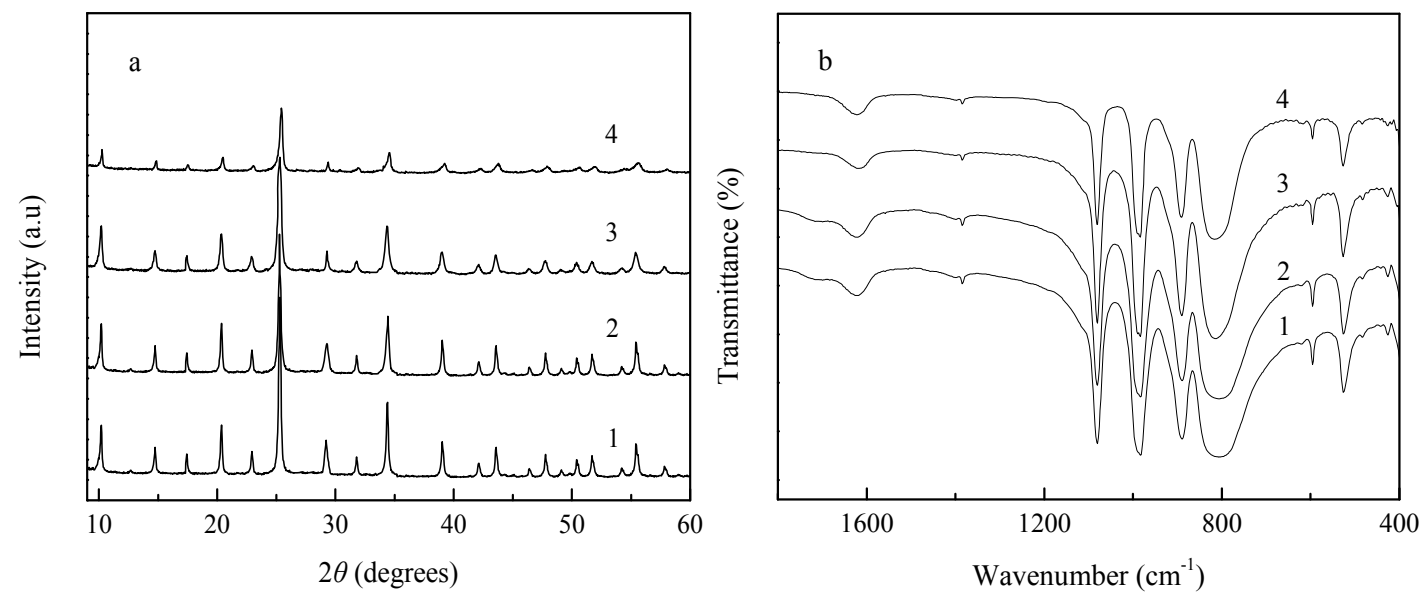

Figure 3. XRD patterns (a) and FT-IR spectra (b) of HPW, K-HPW-1, K-HPW-2, and K-HPW-3. 1. HPW; 2. K-HPW-1; 3. K-HPW-2; 4. K-HPW-3.

HPW, K-HPW-1, K-HPW-2, and K-HPW-3 were further characterized by FT-IR, and the results are shown in Figure $3 \mathrm{~b}$. As reported that Keggin structure is composed of a $\mathrm{PO}_{4}$ tetrahedron, surrounded by four $\mathrm{W}_{3} \mathrm{O}_{9}$ groups formed by edge sharing octahedral [23]. The FT-IR spectra of the catalysts shows characteristic peaks of Keggin structure in the range from 700 to $1200 \mathrm{~cm}^{-1}$ in Figure 3. Four types of atoms in keggin are the asymmetry vibrations $\mathrm{P}-\mathrm{O}_{\mathrm{a}}\left(1080 \mathrm{~cm}^{-1}\right), \mathrm{W}-\mathrm{O}_{\mathrm{d}}\left(983 \mathrm{~cm}^{-1}\right), \mathrm{W}-\mathrm{O}_{\mathrm{b}}\left(889 \mathrm{~cm}^{-1}\right)$, 
and $\mathrm{W}-\mathrm{O}_{\mathrm{c}}\left(808 \mathrm{~cm}^{-1}\right)$, respectively, which coincides with those referred in the literature [24]. The results further indicated that Keggin structure remained in case of introduction of potassium.

\subsection{Effects of Reaction Parameters on Ethyl Levulinate Yield}

Effect of reaction temperature varying from 130 to $180{ }^{\circ} \mathrm{C}$ on the fructose conversion and product distributions catalyzed by K-HPW-1 is shown in Figure 4a. It can be seen that almost all the fructose has been converted in the process. Meanwhile, ethyl levulinate yield gradually increased with the increase of reaction temperature from 130 to $150{ }^{\circ} \mathrm{C}$. When the reaction temperature was above $150{ }^{\circ} \mathrm{C}$, ethyl levulinate yield began to decrease. This result suggested that higher temperature is unfavorable for alcoholysis of fructose to ethyl levulinate, which is in accordance with our previous report [14]. Moreover, more humins can be observed as the reaction temperature is increased, which means unwanted side reactions were enhanced in the process. Thus, the optimum temperature for ethyl levulinate formation from fructose was $150{ }^{\circ} \mathrm{C}$ in this study. In addition, 5-ethoxymethylfurfural and levulinic acid can be detected in the reaction liquid. 5-ethoxymethylfurfural is considered as the intermediate in the formation of ethyl levulinate via cleavage of the aldehyde group on the furfural intermediate, and levulinic acid is the end product of the hydrolysis of 5-hydroxymethylfurfural. Both of them are considered as main products in the reaction process. As shown in Figure 4a, the yield of 5-ethoxymethylfurfural was obviously lower, which means most of them were converted to ethyl levulinate and other products. Moreover, the yield of 5-ethoxymethylfurfural decreased from 8.2 to $1.4 \mathrm{~mol} \%$ when the temperature increased from 130 to $180{ }^{\circ} \mathrm{C}$, suggesting that high temperature is unfavorable for the production of 5-ethoxymethylfurfural. Meanwhile, the levulinic acid yield was also very low (less than $3.5 \mathrm{~mol} \%$ ) at different temperature. This result suggested that levulinic acid cannot be produced efficiently in ethanol medium.

Effect of catalyst amount ranging from 0.1 to $1.0 \mathrm{~g}$ on ethyl levulinate yield was further studied, and the catalyst amount also showed significant effect on ethyl levulinate yield (Figure 4b). It can be seen that ethyl levulinate yield increased from 34.9 to $64.6 \mathrm{~mol} \%$ when the catalyst amount was increased from 0.1 to $0.6 \mathrm{~g}$. However, ethyl levulinate yield gradually decreased when the catalyst amount was more than $0.6 \mathrm{~g}$, indicating that the increase of catalyst amount cannot improve the ethyl levulinate yield efficiently. The reason may be more catalysts can enhance the side reactions of the process, which results in the decrease of the ethyl levulinate yield. In the experiments, $0.6 \mathrm{~g}$ of K-HPW-1 was found to be an ideal amount for the synthesis of ethyl levulinate from fructose in ethanol. Meanwhile, 5-ethoxymethylfurfural yield decreased gradually with the increase of catalyst amount, and levulinic acid yield was almost kept in a low level.

Figure $4 \mathrm{c}$ showed the effect of reaction time in terms of the direct synthesis of ethyl levulinate from fructose in ethanol at $150{ }^{\circ} \mathrm{C}$. The ethyl levulinate yield increased during the first $2 \mathrm{~h}$ of the reaction process, and then leveled off after $2 \mathrm{~h}$. Meanwhile, the amount of insoluble humins augmented with the increase of the reaction time. Therefore, $2 \mathrm{~h}$ was taken as the optimal reaction time for ethyl levulinate production. The yield of 5-ethoxymethylfurfural was $8.2 \mathrm{~mol} \%$ at $0 \mathrm{~h}$, and then decreased with the prolonging of the reaction time. This is because longer reaction time can result in further conversion of 5-ethoxymethylfurfural to ethyl levulinate [25]. Similarly, the yield of levulinic acid was very low in the 
whole process. Therefore, it can be concluded the catalytic system is not adaptable for the synthesis of levulinic acid.
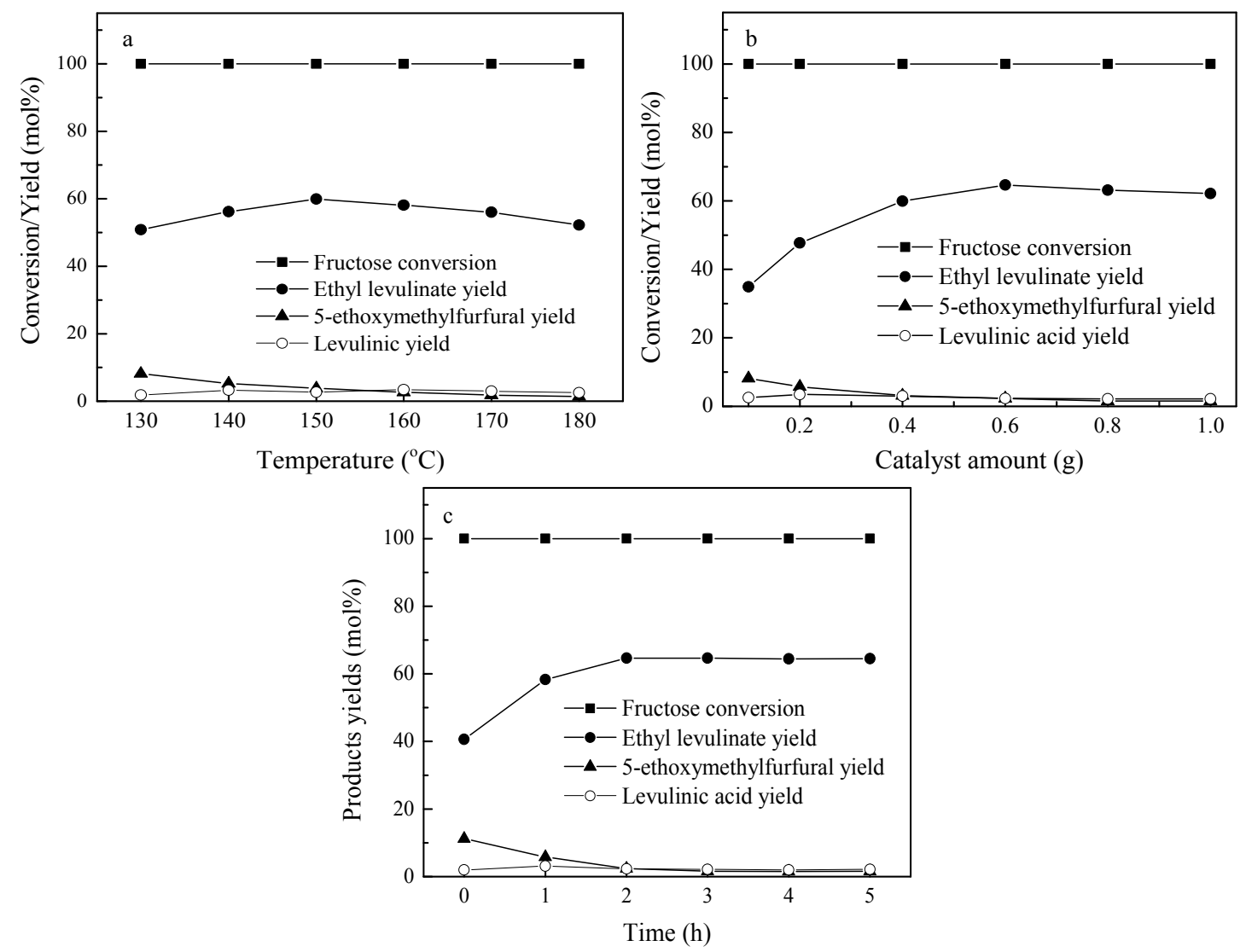

Figure 4. Effect of reaction parameters on product yields. (a) effect of temperature. Reaction conditions: fructose $0.8 \mathrm{~g}, \mathrm{~K}-\mathrm{HPW}-10.4 \mathrm{~g}$, ethanol $40 \mathrm{~mL}$, reaction time $2 \mathrm{~h}$; (b) effect of catalyst amount. Reaction conditions: fructose $0.8 \mathrm{~g}$, ethanol $40 \mathrm{~mL}$, temperature $150{ }^{\circ} \mathrm{C}$, reaction time $2 \mathrm{~h}$; (c) effect of reaction time. Reaction conditions: fructose $0.8 \mathrm{~g}$, K-HPW-1 $0.6 \mathrm{~g}$, ethanol $40 \mathrm{~mL}$, temperature $150{ }^{\circ} \mathrm{C}$.

In order to obtain the optimal process conditions for alcoholysis of fructose to ethyl levulinate, an orthogonal experiment was further carried out based on the discussion above. The arrangements of orthogonal experiments with three factors (temperature, catalyst amount, and time) and the analysis results are listed in Table 1. It can be seen that the maximum ethyl levulinate yield is $64.6 \mathrm{~mol} \%$ under the condition of temperature $150{ }^{\circ} \mathrm{C}$, time $2 \mathrm{~h}$, and catalyst amount $0.6 \mathrm{~g}$, while the lowest ethyl levulinate is $42.3 \mathrm{~mol} \%$ for temperature $140{ }^{\circ} \mathrm{C}$, time $1 \mathrm{~h}$, and catalyst amount $0.4 \mathrm{~g}$. The most significant influencing among the three factors on ethyl levulinate yield was found to be temperature, followed by time, and catalyst amount. To obtain each factor, the intuitive analyses were performed as shown in Figure 5, based on statistical calculation using the data in Table 1. In summary, the optimization results for high ethyl levulinate production were temperature of $150^{\circ} \mathrm{C}$, time of $2 \mathrm{~h}$, and catalyst amount of $0.6 \mathrm{~g}$. 
Table 1. The results of orthogonal experiments.

\begin{tabular}{ccccc}
\hline Entry & Temperature $\left({ }^{\circ} \mathbf{C}\right)$ & Catalyst Amount (g) & Time (h) & Ethyl Levulinate Yield (mol\%) \\
\hline 1 & 140 & 0.4 & 1.0 & 42.3 \\
2 & 140 & 0.6 & 1.5 & 52.5 \\
3 & 140 & 0.8 & 2.0 & 53.1 \\
4 & 150 & 0.4 & 1.5 & 55.8 \\
5 & 150 & 0.6 & 2.0 & 64.6 \\
6 & 150 & 0.8 & 1.0 & 54.3 \\
7 & 160 & 0.4 & 2.0 & 58.1 \\
8 & 160 & 0.6 & 1.0 & 54.6 \\
9 & 160 & 0.8 & 1.5 & 57.8 \\
$k_{1}$ & 49.3 & 52.1 & 50.4 & - \\
$k_{2}$ & 58.2 & 57.2 & 55.4 & - \\
$k_{3}$ & 56.8 & 55.1 & 58.6 & - \\
Range & 8.9 & 5.1 & 8.2 & - \\
\hline
\end{tabular}

Note: $k_{i}$, the mean of ethyl levulinate yield at each level; Range $=k_{\max }-k_{\min }$.

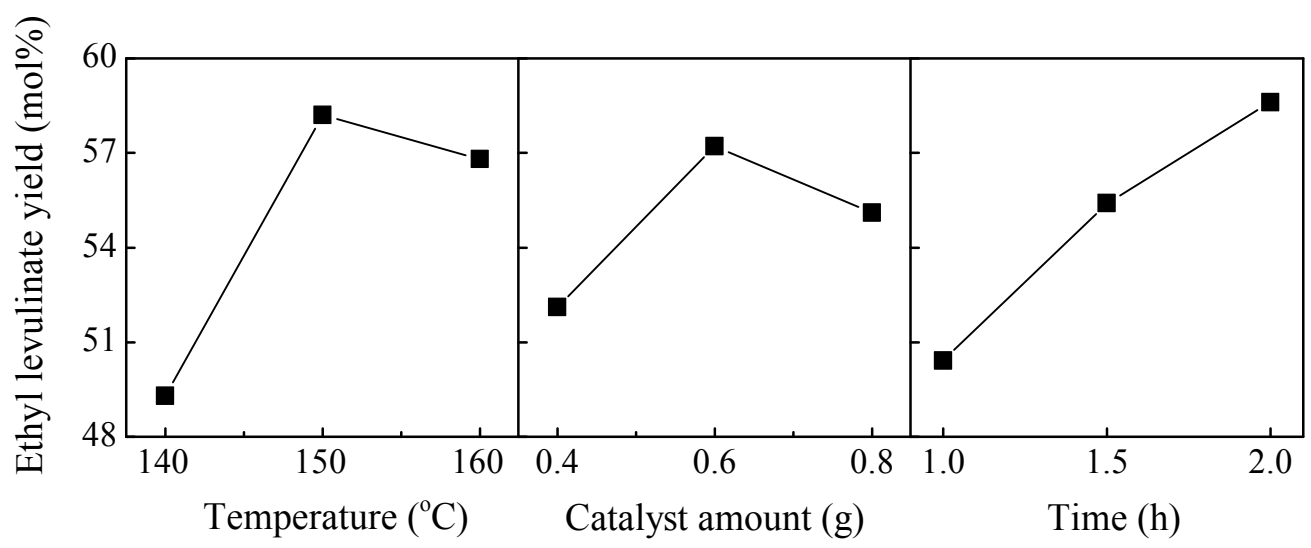

Figure 5. Intuitive analysis of the relationship between process conditions (temperature, catalyst amount, and time) and ethyl levulinate yield.

\subsection{Effect of Co-Solvents on Synthesis of Ethyl Levulinate from Fructose}

To investigate the effect of low-polar organic solvents on ethyl levulinate yield, six organic solvents including n-hexane, toluene, methyl isobutyl ketone (MIBK), acetonitrile, tetrahydrofuran (THF), and 1,4-dioxane were added as co-solvent in ethanol medium, and the results are shown in Figure 6a. It was reported that the introduction of low-polar co-solvent in ethanol may improve the ethyl levulinate yield [26]. However, different solvents show different effects on the yield. As shown in Figure 6a, the addition of $n$-hexane, THF, and 1,4-Dioxane resulted in slightly decreased yield. In comparison, the yield of ethyl levulinate was the lowest when acetonitrile was used as co-solvent, which has the highest polarity. Clearly, toluene showed the best performance on the selectivity of ethyl levulinate with a yield of $61.1 \mathrm{~mol} \%$. This is most likely because toluene acted as an effective extracting agent for separating ethyl levulinate and avoiding its further degradation [27]. Thus, toluene can be chosen as a suitable co-solvent in the following exploration. 

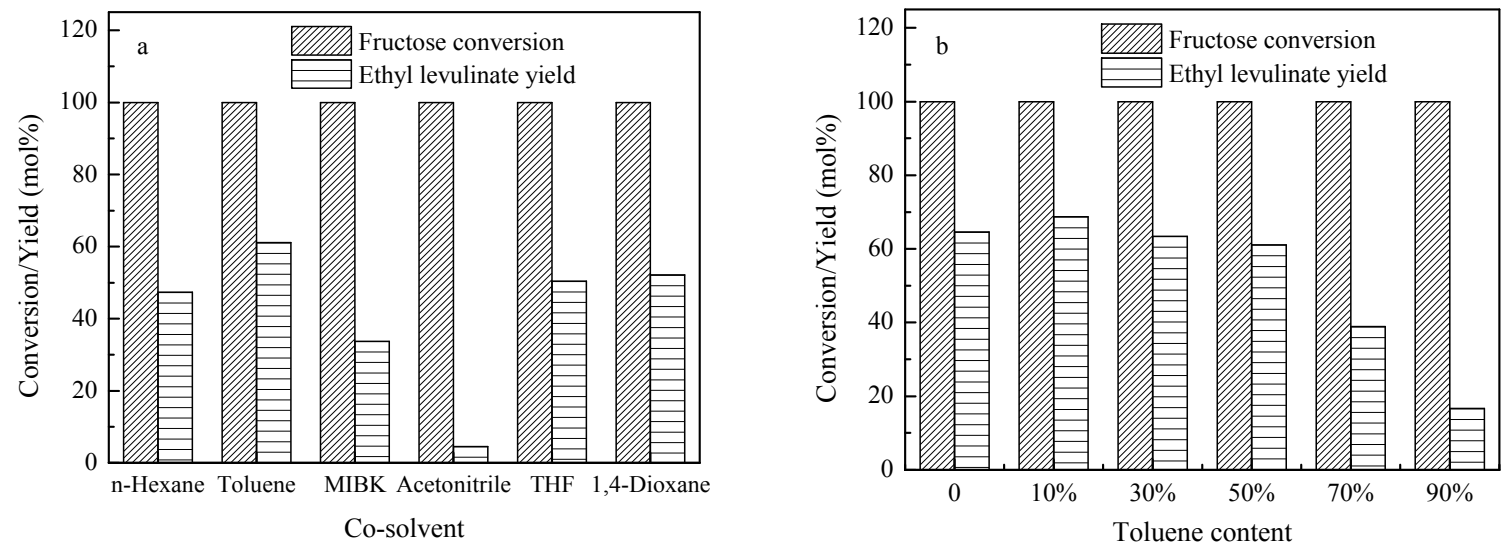

Figure 6. Effect of co-solvents (a) and toluene content (b) on synthesis of ethyl levulinate from fructose. Reaction conditions: fructose $0.8 \mathrm{~g}, \mathrm{~K}-\mathrm{HPW}-10.6 \mathrm{~g}$, temperature $150{ }^{\circ} \mathrm{C}$, reaction time $2 \mathrm{~h}$, ethanol $20 \mathrm{~mL}$, co-solvent $20 \mathrm{~mL}$ (a) or ethanol/toluene $40 \mathrm{~mL}$ (b).

On this basis, effect of toluene content on the synthesis of ethyl levulinate from fructose was further studied and the results are shown in Figure $6 \mathrm{~b}$. When the toluene content was $10 \%$, the yield of ethyl levulinate in the ethanol/toluene media was $68.7 \mathrm{~mol} \%$, which was higher than that of $64.6 \mathrm{~mol} \%$ in pure ethanol media. In this reaction, $10 \%$ volume fraction of toluene may protect ethyl levulinate and suppress the side reactions during the reaction process. However, the yield of ethyl levulinate decreased with further increase of the toluene content, and it was found that the formation of humins was also increased. Therefore, $10 \%$ volume fraction of toluene can be chosen as the optimum content.

\subsection{Catalyst Recycling}

Recycling experiments for K-HPW-1 were carried out for the conversion of fructose to ethyl levulinate in ethanol/toluene medium under identical experimental conditions. Figure 7 shows the results from recycling K-HPW-1 for five consecutive runs. In the first run, the yield of ethyl levulinate was $68.7 \mathrm{~mol} \%$. With the increasing numbers of catalyst recycling, the yield of ethyl levulinate decreased gradually. After being used for five runs, the yield of ethyl levulinate was still above $50 \mathrm{~mol} \%$, indicating K-HPW-1 has a long lifetime for the conversion of fructose.

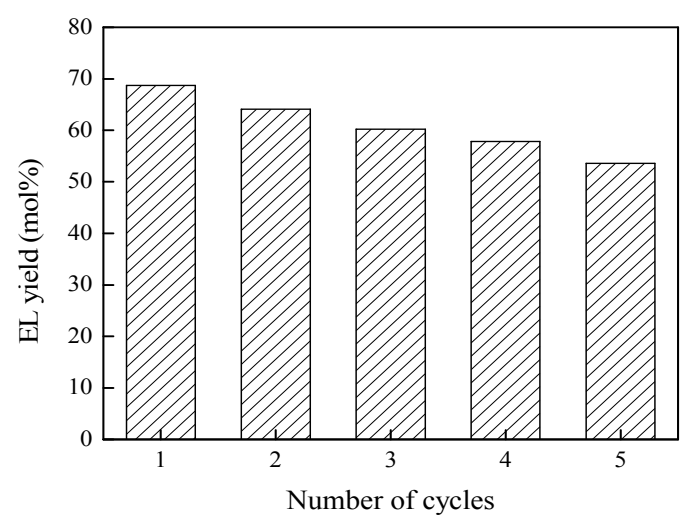

Figure 7. Catalyst recycling experiments with the insoluble K-HPW-1 catalyst. Reaction conditions: fructose $0.8 \mathrm{~g}, \mathrm{~K}-\mathrm{HPW}-10.6 \mathrm{~g}$, toluene $4 \mathrm{~mL}$, ethanol $36 \mathrm{~mL}$, temperature $150{ }^{\circ} \mathrm{C}$, reaction time $2 \mathrm{~h}$. 
The surface structures of fresh and recycled K-HPW-1 catalysts were characterized by XRD, FT-IR and SEM, respectively. In Figure 8, characteristic diffraction peaks of HPW on the recycled catalyst still appeared at $2 \theta=10.2^{\circ}, 21.3^{\circ}, 25.3^{\circ}$, and $34.4^{\circ}$. Figure $8 \mathrm{~b}$ also show the characteristic bands of HPW in the catalysts were observed at 1080, 992, 889, and $808 \mathrm{~cm}^{-1}$ in Figure $8 \mathrm{~b}$. Both of them indicated the Keggin structure remained in the recycled catalyst K-HPW-1. Besides, the surface morphologies of the fresh and recycled K-HPW-1 catalysts were also observed by SEM, and the results are shown in Figure 9. Figure 9a presented the distinguishable and homogeneous morphology of the fresh K-HPW-1 catalyst at the levels of 20,000 times magnification, and Figure 9b showed a ruleless and fuzzy morphology of the recycled catalyst after five runs. Besides, the color of the catalyst was changed from white to dark gray. These morphology and color differences are attributed to the carbon deposition caused by degradation of products.
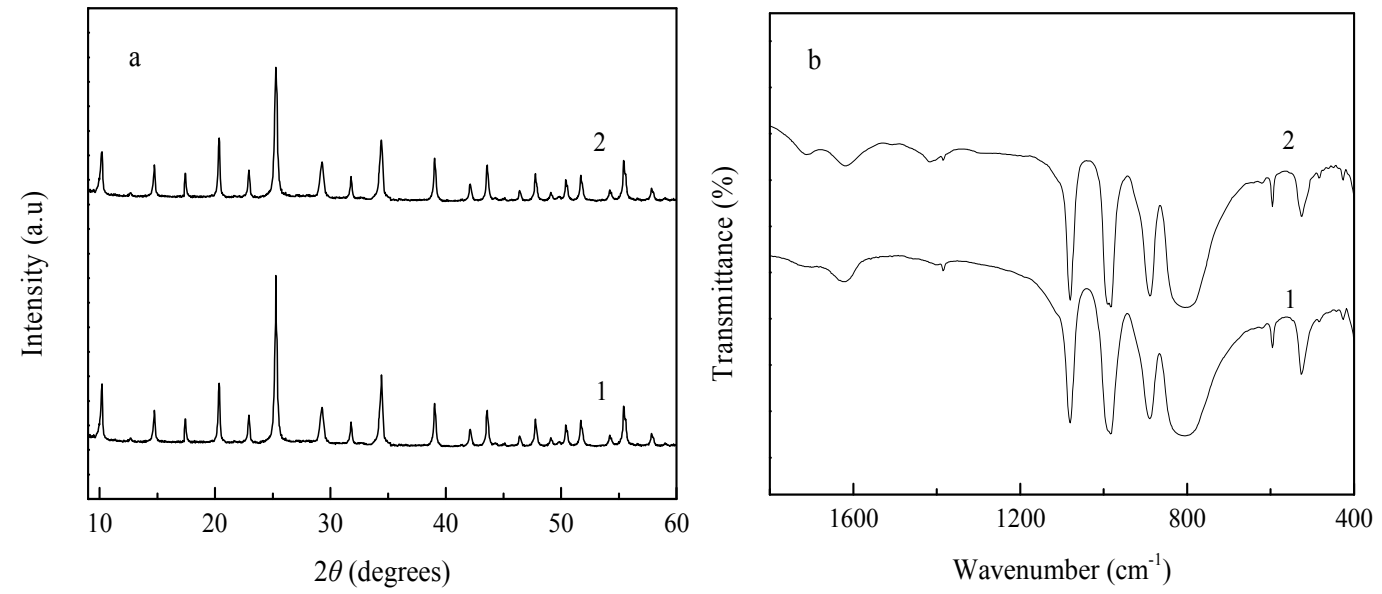

Figure 8. XRD patterns (a) and FT-IR spectra (b) of fresh and recycled K-HPW-1. 1. Fresh catalyst; 2 . Recycled catalyst after five runs.

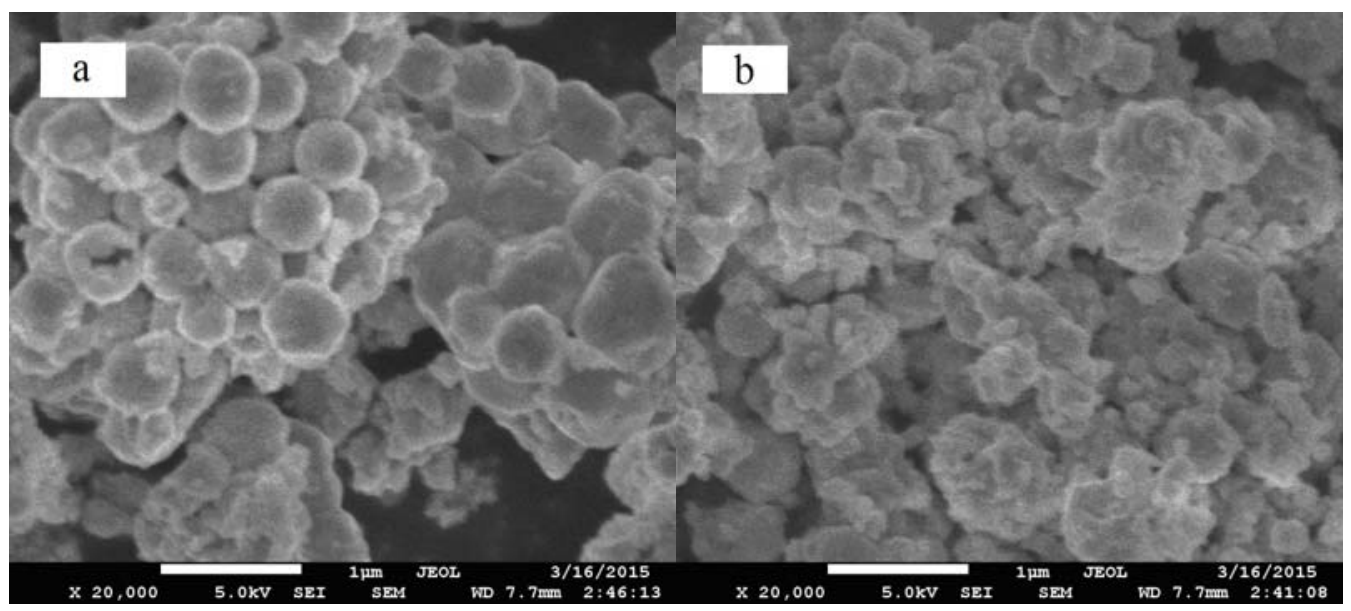

Figure 9. SEM images for fresh catalyst (a) and recycled catalyst after five runs (b).

\subsection{Effect of Various Raw Materials on Synthesis of Ethyl Levulinate}

In order to evaluate the generality of the catalyst for the synthesis of ethyl levulinate, the insoluble K-HPW-1 catalyst was further extended to the other carbohydrates such as glucose, sucrose, inulin, and 
cellulose. As shown in Table 2. Fructose gave the highest yield of ethyl levulinate, while the yield of ethyl levulinate converted from glucose was only $14.5 \mathrm{~mol} \%$, which was far lower than that of fructose. Sucrose and inulin are two of the most abundant and renewable saccharides from biomass. When sucrose was used, a medium ethyl levulinate yield of $35.4 \mathrm{~mol} \%$ was obtained. Whereas, $52.3 \mathrm{~mol} \%$ of ethyl levulinate yield was obtained for inulin which consists of one molecule of glucose and 1-59 molecules of fructose. Furthermore, the yield of ethyl levulinate from cellulose consisted of glucose units, was $14.8 \mathrm{~mol} \%$ at $220{ }^{\circ} \mathrm{C}$. The results demonstrated that K-HPW-1 was an effective catalyst in terms of synthesis of ethyl levulinate from fructose units in carbohydrates.

Table 2. Effect of various raw materials on synthesis of ethyl levulinate.

\begin{tabular}{cccccc}
\hline $\begin{array}{c}\text { Raw } \\
\text { Meterials }\end{array}$ & $\begin{array}{c}\text { Temperature } \\
\left({ }^{\circ} \mathbf{C}\right)\end{array}$ & $\begin{array}{c}\text { Conversion } \\
\mathbf{( \% )}\end{array}$ & $\begin{array}{c}\text { Ethyl Levulinate } \\
\text { Yield (mol\%) }\end{array}$ & $\begin{array}{c}\text { 5-Ethoxymethylfur-fural } \\
\text { Yield (mol\%) }\end{array}$ & $\begin{array}{c}\text { Levulinic Acid } \\
\text { Yield (mol\%) }\end{array}$ \\
\hline Fructose & 150 & 100 & 68.7 & 3.5 & 2.1 \\
Glucose & 150 & 99.2 & 14.5 & 1.5 & 1.7 \\
Sucrose & 150 & 98.1 & 35.4 & 2.1 & 2.0 \\
Inulin & 150 & $>95$ & 52.3 & 2.5 & 2.1 \\
Cellulose & 220 & $>95$ & 14.8 & 1.2 & 1.4 \\
\hline
\end{tabular}

Reaction conditions: carbohydrate 0.8 g, K-HPW-1 0.6 g, toluene $4 \mathrm{~mL}$, ethanol $36 \mathrm{~mL}$, reaction time $2 \mathrm{~h}$.

For comparison, several related results about ethyl levulinate production from fructose catalyzed by different catalysts are shown in Table 3 . The yields of ethyl levulinate were observably different due to the difference of the catalysts. Besides, the yields also depended on the reaction conditions. Saravanamurugan et al. [28] and Chen et al. [29] reported that high ethyl levulinate yields can be obtained with ionic liquids as catalysts, while it took longer time (more than $12 \mathrm{~h}$ ) to reach the reaction equilibrium and the cost of catalysts was also quite high. In most studies, the yield of ethyl levulinate was lower than $60 \mathrm{~mol} \%$. However, the yield of ethyl levulinate can reach up to $68.7 \mathrm{~mol} \%$ under the optimum conditions in this study, which means K-HPW-1 is an efficient catalyst for synthesis of ethyl levulinate from fructose.

Table 3. Comparison of different research on ethyl levulinate production from fructose.

\begin{tabular}{|c|c|c|c|c|}
\hline Research & Catalyst & Medium & Reaction Conditions & $\begin{array}{c}\text { Ethyl Levulinate } \\
\text { Yield (mol\%) }\end{array}$ \\
\hline$[9]$ & Amberlyst 70 & Ethanol/water & $175^{\circ} \mathrm{C} 2 \mathrm{~h}$ & 37 \\
\hline$[15]$ & $\mathrm{SO}_{4}^{2-} / \mathrm{TiO}_{2}$ & Ethanol & $200{ }^{\circ} \mathrm{C} 2 \mathrm{~h}$ & 59 \\
\hline$[16]$ & H-USY(6) & Ethanol & $150^{\circ} \mathrm{C} 20 \mathrm{~h}$ & 40 \\
\hline$[28]$ & {$\left[\mathrm{NEt}_{3} \mathrm{BSO}_{3} \mathrm{H}\right]\left[\mathrm{HSO}_{4}\right]$} & Ethanol & $140^{\circ} \mathrm{C} 24 \mathrm{~h}$ & 77 \\
\hline [29] & IL-POMs $^{\text {a }}$ & Ethanol & $120^{\circ} \mathrm{C} 12 \mathrm{~h}$ & $28-80$ \\
\hline$[30]$ & $\mathrm{SO}_{3} \mathrm{H}-\mathrm{SBA}-15$ & Ethanol & $140^{\circ} \mathrm{C} 24 \mathrm{~h}$ & 57 \\
\hline This study & K-HPW-1 & Ethanol/toluene & $150^{\circ} \mathrm{C} 2 \mathrm{~h}$ & 68.7 \\
\hline
\end{tabular}




\section{Experimental Section}

\subsection{Materials}

$\mathrm{H}_{3} \mathrm{PW}_{12} \mathrm{O}_{40} \cdot x \mathrm{H}_{2} \mathrm{O}$, anhydrous ethanol and $\mathrm{KCl}$ were purchased from Kermel Chemical Reagent (Tianjin, China), and the $\mathrm{x}$ value of $\mathrm{H}_{3} \mathrm{PW}_{12} \mathrm{O}_{40} \cdot x \mathrm{H}_{2} \mathrm{O}$ was determined to be 10 by thermogravimetric method [31]. 5-hydroxymethylfurfural, levulinic acid, ethyl levulinate, and fructose with the purity of over 99\% were purchased from Aladdin Chemical Reagent (Shanghai, China). 5-ethoxymethylfurfural (97\%) was obtained from Sigma-Aldrich. Other chemicals were purchased from Kermel Chemical Reagent. All reagents were analytical grade and used without further purification.

\subsection{Catalysts Preparation}

The catalysts were prepared according to a reported procedure [30] as follows: an appropriate amount of metal salt $(0.1 \mathrm{~mol} / \mathrm{L})$ aqueous solution was added dropwise into $20 \mathrm{~mL} \mathrm{HPW}(0.08 \mathrm{~mol} / \mathrm{L})$ with vigorous stirring. Then the mixture was further stirred for another $2 \mathrm{~h}$ at room temperature. The catalyst was obtained by filtration and dried at $80{ }^{\circ} \mathrm{C}$ overnight. Finally, the catalyst was calcined at $300{ }^{\circ} \mathrm{C}$ for $4 \mathrm{~h}$. These prepared catalysts are designated as Cs-HPW-1, Cs-HPW-2.5, Ag-HPW-1, K-HPW-1, K-HPW-2, K-HPW-3, and Sn-HPW-0.75 according to the stoichiometry. Fe-HPW-1 was prepared according to Liu's report [20].

\subsection{Catalytic Reaction Procedure}

The experiments of direct synthesis of ethyl levulinate from fructose in ethanol were performed in a $100 \mathrm{~mL}$ cylindrical pressurized reactor. In a typical run, fructose $(0.8 \mathrm{~g})$, ethanol $(40 \mathrm{~mL})$, and a given amount of solid acid catalysts were mixed in the reactor with a rotation speed of $600 \mathrm{rpm}$. Then, the reactor was brought to the desired temperature by external heating. Time zero was recorded when the set temperature was reached. After reaction, the reactor was cooled down in a cool water bath, and then the reaction mixture was diluted with anhydrous ethanol to $100 \mathrm{~mL}$. The liquid was filtered with a $0.22 \mu \mathrm{m}$ syringe filter prior to analysis by gas chromatography and high-performance liquid chromatography (HPLC). To test stability of the catalyst, K-HPW-1 was recycled by centrifugation after the reaction, and the recovered catalyst was washed with water and ethanol three times each and dried at $110^{\circ} \mathrm{C}$ overnight. Then, it was calcined at $300{ }^{\circ} \mathrm{C}$ for $3 \mathrm{~h}$ before being reused in a new experiment under the same conditions.

\subsection{Products Analysis}

Ethyl levulinate was determined by a gas chromatography equipped with an FFAP capillary column $(30 \mathrm{~m} \times 0.32 \mathrm{~mm} \times 0.33 \mu \mathrm{m})$ and flame ionization detector (FID). The amount of ethyl levulinate was calculated using standard curves based on 1-octanol as the internal standard. Fructose, 5-ethoxymethylfurfural and levulinic acid were quantified by HPLC equipped with a Waters 2412 Refractive Index detector (Milford, MA, USA) and a Bio-Rad HPX-87H column (Hercules, CA, USA) $(300 \mathrm{~mm} \times 7.8 \mathrm{~mm})$ at $65{ }^{\circ} \mathrm{C}$ controlled with the column oven. $0.005 \mathrm{M} \mathrm{H}_{2} \mathrm{SO}_{4}$ with a flow rate of 
$0.6 \mathrm{~mL} / \mathrm{min}$ was used as the mobile phase. The content of above components was calculated by external standard calibration curve method.

\subsection{Catalysts Characterization}

X-ray powder diffraction (XRD) patterns of the samples were carried out using a BRUKER X-ray diffractometer (D8 Advance, Bruker, Germany) with a $\mathrm{Cu} \mathrm{K} \alpha$ radiation source operated. Data were collected from $2 \theta$ between $10^{\circ}$ and $80^{\circ}$ with a step of $0.02^{\circ}$ at a scanning speed of $3 \% \mathrm{~min}$. Fourier transform infrared (FT-IR) spectra were conducted on a spectrometer (Nicolet 6700, Thermo Scientific, Waltham, MA, USA) at a spectral resolution of $4 \mathrm{~cm}^{-1}$ in the wave number range of 400 to $4000 \mathrm{~cm}^{-1}$. A scanning electron microscope (SEM) (JSM-7500F, JEOL, Tokyo, Japan) was used to observe the surface morphology of the catalysts.

\section{Conclusions}

The $\mathrm{H}^{+}$of HPW substituted by $\mathrm{K}^{+}$could modify the acidity of HPW and form an efficient catalyst $\mathrm{K}-\mathrm{HPW}-1$ for synthesis of ethyl levulinate from fructose. The highest ethyl levulinate yield of $64.6 \mathrm{~mol} \%$ was obtained at $150{ }^{\circ} \mathrm{C}$ within $2 \mathrm{~h}$ in ethanol. Six organic solvents were tested as co-solvents, and it was found toluene showed the best performance on the selectivity of ethyl levulinate. The introduction of toluene can improve the yield of ethyl levulinate to $68.7 \mathrm{~mol} \%$. Moreover, the recovered catalyst retained high activity with the yield of ethyl levulinate converted from fructose above $50 \mathrm{~mol} \%$ after being used in five runs. The comparative research also showed K-HPW-1 is an efficient catalyst for synthesis of ethyl levulinate from fructose.

\section{Acknowledgments}

This work was financially supported by the National Natural Science Foundation of China (No.21176227) and the State Key Laboratory of Motor Vehicle Biofuel Technology (KFKT2014008).

\section{Author Contributions}

Chun Chang, Guizhuan $\mathrm{Xu}$, and Shiqiang Zhao contributed to the experimental design. Shiqiang Zhao, Shuqi Fang, Fengguang Du, and Ze Liu contributed to all the experimental data collection. Shiqiang Zhao wrote the first draft of the manuscript that was then extensively improved by Chun Chang and Guizhuan Xu.

\section{Conflicts of Interest}

The authors declare no conflict of interest.

\section{References}

1. Chatterjee, C.; Pong, F.; Sen, A. Chemical conversion pathways for carbohydrates. Green Chem. 2015, 17, 40-71. 
2. Mielenz, J.R.; Rodriguez, M.; Thompson, O.A.; Yang, X.; Yin, H. Development of Agave as a dedicated biomass source: Production of biofuels from whole plants. Biotechnol. Biofuels 2015, 8, 79.

3. Bozell, J.J.; Moens, L.; Elliott, D.C.; Wang, Y.; Neuenscwander, G.G.; Fitzpatrick, S.W.; Bilski, R.J.; Jarnefeld, J.L. Production of levulinic acid and use as a platform chemical for derived products. Resour. Conserv. Recycl. 2000, 28, 227-239.

4. Chang, C.; Cen, P.; Ma, X. Levulinic acid production from wheat straw. Bioresour. Technol. 2007, $98,1448-1453$.

5. Verevkin, S.P.; Emel'yanenko, V.N. Renewable platform-chemicals and materials: Thermochemical study of levulinic acid. J. Chem. Thermodyn. 2012, 46, 94-98.

6. Cha, J.Y.; Hanna, M.A. Levulinic acid production based on extrusion and pressurized batch reaction. Ind. Crops Prod. 2002, 16, 109-118.

7. Chang, C.; Ma, X.; Cen, P. Kinetics of Levulinic Acid Formation from Glucose Decomposition at High Temperature1. Chin. J. Chem. Eng. 2006, 14, 708-712.

8. Girisuta, B.; Janssen, L.P.B.M.; Heeres, H.J. Kinetic Study on the Acid-Catalyzed Hydrolysis of Cellulose to Levulinic Acid. Ind. Eng. Chem. Res. 2007, 46, 1696-1708.

9. Hu, X.; Wang, S.; Westerhof, R.J.M.; Wu, L.; Song, Y.; Dong, D.; Li, C.Z. Acid-catalyzed conversion of C6 sugar monomer/oligomers to levulinic acid in water, tetrahydrofuran and toluene: Importance of the solvent polarity. Fuel 2015, 141, 56-63.

10. Weingarten, R.; Conner, W.C.; Huber, G.W. Production of levulinic acid from cellulose by hydrothermal decomposition combined with aqueous phase dehydration with a solid acid catalyst. Energy Environ. Sci. 2012, 5, 7559-7574.

11. Christensen, E.; Williams, A.; Paul, S.; Burton, S.; McCormick, R.L. Properties and performance of levulinate esters as diesel blend components. Energy Fuels 2011, 25, 5422-5428.

12. Climent, M.J.; Corma, A.; Iborra, S. Conversion of biomass platform molecules into fuel additives and liquid hydrocarbon fuels. Green Chem. 2014, 16, 516-547.

13. Neves, P.; Lima, S.; Pillinger, M.; Rocha, S.M.; Rocha, J.; Valente, A.A. Conversion of furfuryl alcohol to ethyl levulinate using porous aluminosilicate acid catalysts. Catal. Today 2013, 218-219, 76-84.

14. Chang, C.; Xu, G.; Jiang, X. Production of ethyl levulinate by direct conversion of wheat straw in ethanol media. Bioresour. Technol. 2012, 121, 93-99.

15. Peng, L.C.; Lin, L.; Zhang, J., Shi, J., Liu, S. Solid acid catalyzed glucose conversion to ethyl levulinate. Appl. Catal. A 2011, 397, 259-265.

16. Saravanamurugan, S.; Riisager, A. Zeolite catalyzed transformation of carbohydrates to alkyl levulinates. ChemCatChem 2013, 5, 1754-1757.

17. Zhao, S.; Xu, G.; Chang, J.; Chang, C.; Bai, J.; Fang, S.; Liu, Z. Direct Production of Ethyl Levulinate from Carbohydrates Catalyzed by H-ZSM-5 Supported Phosphotungstic Acid. Bioresource 2015, 10, 2223-2234.

18. Chang, C.; Xu, G.; Zhu, W.; Bai, J.; Fang, S. One-pot production of a liquid biofuel candidateEthyl levulinate from glucose and furfural residues using a combination of extremely low sulfuric acid and zeolite USY. Fuel 2015, 140, 365-370. 
19. Costa, V.V.; da Silva Rocha, K.A.; Kozhevnikov, I.V.; Kozhevnikova, E.F.; Gusevskaya, E.V. Heteropoly acid catalysts for the synthesis of fragrance compounds from biorenewables: Isomerization of limonene oxide. Catal. Sci. Technol. 2013, 3, 244-250.

20. Liu, Y.; Liu, C.-L.; Wu, H.-Z.; Dong, W.-S. An Efficient Catalyst for the Conversion of Fructose into Methyl Levulinate. Catal. Lett. 2013, 143, 1346-1353.

21. Guo, Y.; Hu, C. Heterogeneous photocatalysis by solid polyoxometalates. J. Mol. Catal. A 2007, 262, 136-148.

22. Hernández-Cortez, J.G.; Martinez, L.; Soto, L.; López, A.; Navarrete, J.; Manríquez, M.; Lara, V.H.; López-Salinas, E. Liquid phase alkylation of benzene with dec-1-ene catalyzed on supported 12-tungstophosphoric acid. Catal. Today 2010, 150, 346-352.

23. Chai, F.; Cao, F.; Zhai, F.; Chen, Y.; Wang, X.; Su, Z. Transesterification of Vegetable Oil to Biodiesel using a Heteropolyacid Solid Catalyst. Adv. Synth. Catal. 2007, 349, 1057-1065.

24. Ghanbari-Siahkali, A.; Philippou, A.; Dwyer, J.; Anderson, M.W. The acidity and catalytic activity of heteropoly acid on MCM-41 investigated by MAS NMR, FTIR and catalytic tests. Appl. Catal. A 2000, 192, 57-69.

25. Yang, Y.; Abu-Omar, M.M.; Hu, C. Heteropolyacid catalyzed conversion of fructose, sucrose, and inulin to 5-ethoxymethylfurfural, a liquid biofuel candidate. Appl. Energy 2012, 99, 80-84.

26. Garves, K. Acid Catalyzed Degradation of Cellulose in Alcohols. J. Wood Chem. Technol. 1988, 8, 121-134.

27. Tang, X.; Sun, Y.; Zeng, X.; Hao, W.; Lin, L.; Liu, S. Novel Process for the Extraction of Ethyl Levulinate by Toluene with Less Humins from the Ethanolysis Products of Carbohydrates. Energy Fuels 2014, 28, 4251-4255.

28. Saravanamurugan, S.; Nguyen van Buu, O.; Riisager, A. Conversion of Mono- and Disaccharides to Ethyl Levulinate and Ethyl Pyranoside with Sulfonic Acid-Functionalized Ionic Liquids. ChemSusChem 2011, 4, 723-726.

29. Chen, J.; Zhao, G.; Chen, L. Efficient production of 5-hydroxymethylfurfural and alkyl levulinate from biomass carbohydrate using ionic liquid-based polyoxometalate salts. RSC $A d v$. 2014, 4, 4194-4202.

30. Saravanamurugan, S.; Riisager, A. Solid acid catalysed formation of ethyl levulinate and ethyl glucopyranoside from mono- and disaccharides. Catal. Commun. 2012, 17, 71-75.

31. Ren, Y.; Liu, B.; Zhang, Z.; Lin, J. Silver-exchanged heteropolyacid catalyst $\left(\mathrm{Ag}_{1} \mathrm{H}_{2} \mathrm{PW}\right)$ : An efficient heterogeneous catalyst for the synthesis of 5-ethoxymethylfurfural from 5-hydroxymethylfurfural and fructose. J. Ind. Eng. Chem. 2015, 21, 1127-1131.

(C) 2015 by the authors; licensee MDPI, Basel, Switzerland. This article is an open access article distributed under the terms and conditions of the Creative Commons Attribution license (http://creativecommons.org/licenses/by/4.0/). 